\title{
Value of appendicular skeletal muscle mass to total body fat ratio in predicting obesity in elderly people: a 2.2-year longitudinal study
}

Yu-Jie Zhang ${ }^{1,2+}$, Shi-Hui Fu ${ }^{2 \dagger}$, Jing-Xin Wang ${ }^{3,4}$, Xin Zhao ${ }^{5}$, Yao Yao ${ }^{6,7}$ and Xiao-Ying Li ${ }^{2 *}$

\begin{abstract}
Background: Obesity is a disease characterized by much fat accumulation and abnormal distribution, which was related to cardiovascular diseases, diabetes mellitus (DM) and muscular skeletal diseases. The aim of this study was to evaluate the usefulness of appendicular skeletal muscle mass to total body fat ratio (ASM/TBF) in screening for the risk of obesity in elderly people.

Methods: A prospective study was carried out with 446 participants (non-obese elderly people with body mass index (BMI) $<28 \mathrm{~kg} / \mathrm{m}^{2}$ ) who underwent baseline and an average around 2.2-year follow-up health check-up examinations.

Results: The mean age at baseline was 63.6 years. The incidence of new obesity was $5.4 \%$ during follow-up. Linear regression demonstrated that baseline ASM/TBFs were negatively correlated with follow-up BMls in both men and women $(\beta=-1.147$ (-1.463--0.831) for men and -4.727 (-5.761-3.692) for women). The cut-off points of baseline ASM/TBF in elderly people for obesity were 1.24 in men and 0.90 in women which were identified by Classification and Regression Tree (CART). Logistic regression showed that both men and women with decreased ASM/TBF had higher risks of obesity over the follow-up period (Relative Risk (RRs) $=5.664(1.879-17.074)$ for men and 34.856 (3.930-309.153) for women).
\end{abstract}

Conclusions: Elderly people with a low ASM/TBF had a higher risk of new obesity, which suggested that ASM/TBF should be considered in obesity management in the elderly.

Keywords: Appendicular skeletal muscle mass, Elderly, Obesity, Total body fat

\section{Background}

Obesity is a disease characterized by much fat accumulation and abnormal distribution, which is related to cardiovascular diseases, diabetes mellitus (DM) and muscular skeletal diseases [1]. Severe obesity has been proved to be

\footnotetext{
* Correspondence: xyli3012@163.com

${ }^{\dagger}$ Yu-Jie Zhang and Shi-Hui Fu contributed equally to this work.

2Department of Geriatric Cardiology, Chinese PLA General Hospital, Beijing, China

Full list of author information is available at the end of the article
}

a risk factor for shortened life expectancy and poor prognosis [2]. As the population is aging, obesity is becoming more prevalent among elderly people [3]. Despite body mass index (BMI) can somehow reflect integral fatness among individuals, it may make false estimating over adiposity because of fat deposition in viscera or not $[4,5]$.

The close relationship between appendicular skeletal muscle mass (ASM) / total body fat (TBF) and sarcopenia obesity, which was defined by top TBF quartile, has been well recognized in previous studies [6]. However,

(C) The Author(s). 2020 Open Access This article is licensed under a Creative Commons Attribution 4.0 International License, which permits use, sharing, adaptation, distribution and reproduction in any medium or format, as long as you give appropriate credit to the original author(s) and the source, provide a link to the Creative Commons licence, and indicate if changes were made. The images or other third party material in this article are included in the article's Creative Commons licence, unless indicated otherwise in a credit line to the material. If material is not included in the article's Creative Commons licence and your intended use is not permitted by statutory regulation or exceeds the permitted use, you will need to obtain permission directly from the copyright holder. To view a copy of this licence, visit http://creativecommons.org/licenses/by/4.0/. The Creative Commons Public Domain Dedication waiver (http://creativecommons.org/publicdomain/zero/1.0/) applies to the data made available in this article, unless otherwise stated in a credit line to the data. 
the association between this ratio and new obesity has not been adequately investigated. Besides, it is still not known whether ASM/TBF has significant value to access the risk of obesity.

ASM and TBF, represented muscle and fat components and measured by Bioelectric impedance (BIA) technique, were shown after safe current passed through the human body $[7,8]$. Previous studies indicated that $\mathrm{TBF} /$ weight was a better predictor of cardiovascular risk factors than BMI [7], partly because visceral fat, increasing gradually with aging, was more relevant to TBF. Besides, another study suggested that ASM/TBF was more related to cardio-metabolic and functional abnormalities than TBF/weight, which indicated that ASM/TBF was worth further exploring [9]. However, there was no agreement about the cut-off points for ASM/TBF to diagnose obesity let alone predict it.

Although ASM/TBF has been demonstrated previously [6], little is known about its role in simple obesity assessment. It is necessary to determine whether low ASM/ $\mathrm{TBF}$ is a risk factor for obesity, which may decrease the quality of life in elderly people. Therefore, the aim of the study was to evaluate the usefulness of ASM/TBF in screening for the risk of obesity in elderly people.

\section{Methods \\ Subjects}

Elderly people aged over 60 years who underwent a routine health check-up in Chinese PLA General Hospital from February 2013 to February 2014 were enrolled in this study. The inclusion criteria were elderly people aged over 60 who accepted BIA and anthropometric measurements. Participants were excluded for any of those cases: (a) extremities diseases; (b) previous obesity; (c) diagnosed metastatic advanced tumor or malignant tumor discovery less than 5 years; (d) secondary obesity caused by several diseases, such as Cushing syndrome. Five hundred and forty-five participants were recruited at baseline. Visits were performed at baseline and the next before February 2016. The follow-up time was between $0.6-3.8$ years. Finally, a total of 446 participants completed the average around 2.2 years follow-up. The missing rate was $18.2 \%$. All participants were given written informed consents and the study protocol was approved by the Institutional Review Board of Chinese PLA General Hospital.

\section{Data collection and measurements}

Past history (e.g., obesity) and personal history (e.g., smoking habit) were collected by trained healthcare providers. The method of BIA measurement can be seen from our teams' previous works $[10,11]$. Other anthropometric indicators were on standardized tests [12].

\section{Diagnostic definitions}

The definition of smoking history was based on Report on Cardiovascular Diseases in China [13]. Obesity was defined as $B M I \geq 28 \mathrm{~kg} / \mathrm{m}^{2}$ [14]. The definition of DM was based on Chinese Society of Diabetes (2013 edition) [15].

\section{Statistical analysis}

Data were analyzed by the Statistical Package for Social Sciences, version 22.0 (SPSS, Inc., Chicago, IL, USA). Continuous variables under normal distribution and categorical data were expressed as mean [standard deviation (SD)] and count (percentage), respectively. Classification and Regression Tree (CART) Models, measured in terms of true classification rate of the classifier, were built through a process known as binary recursive partitioning, which is an iterative process that splits the data into partitions or branches, and then continues splitting each partition into smaller groups as the method moves up each branch. The purpose of the analyses via tree-building algorithms was to determine a set of if-then logical (split) conditions that permit accurate prediction or classification of cases. Cut-off values of baseline ASM/TBF for obesity were identified by CART. Moreover, logistic regression was used to examine Relative Risk (RRs) of new obesity in subjects with low ASM/ TBF determined by CART. A two-sided $P$ value $<0.05$ was considered statistically significant.

\section{Results}

\section{Participants' clinical characteristics}

The average age at baseline was 63.6 years. The minimum age was 60 and the maximum age was 81 . The incidence of new obesity was $5.4 \%$ during follow-up. Subjects with new obesity had significantly lower baseline ASM/TBF than subjects without new obesity $(P<$ 0.001 in women and $P=0.119$ in men, Table 1 ).

\section{Effect of baseline ASM/TBF on follow-up BMI}

Linear regression demonstrated that baseline ASM/TBFs were negatively correlated with follow-up body mass indexes (BMIs) in both men and women $(\beta=-1.147$ for men and -4.727 for women even after adjusted for age, albumin and DM history; all $P<0.001$, Table 2).

\section{Cut-off point of baseline ASM/TBF for future obesity}

The cut-off points of baseline ASM/TBF in elderly people for obesity were 1.24 in men and 0.90 in women which were identified by CART. Based on cut-off points, decreased ASM/TBFs were defined as less than 1.24 in men and 0.90 in women (Fig. 1). 
Table 1 Participants' clinical characteristics

\begin{tabular}{|c|c|c|c|c|}
\hline & \multicolumn{2}{|l|}{ Non obesity } & \multicolumn{2}{|l|}{ New obesity } \\
\hline & Male $(n=250)$ & Female $(n=172)$ & Male $(n=15)$ & Female $(n=9)$ \\
\hline Age (years) & $63.8(4.1)$ & $63.5(3.4)$ & $64.4(5.2)$ & $62.4(2.5)$ \\
\hline Smoking history $(n, \%)$ & $146(58.4)$ & $12(7.0)$ & $9(60.0)$ & $1(11.1)$ \\
\hline BMI $\left(\mathrm{kg} / \mathrm{m}^{2}\right)$ & $24.08(1.94)$ & $23.54(2.21)$ & $26.46(0.91)^{* *}$ & $26.32(0.74)^{\dagger+}$ \\
\hline Follow-up BMI (kg/m²) & $24.21(2.00)$ & $23.67(2.14)$ & $27.83(0.41)^{* *}$ & $28.12(0.45)^{+\dagger}$ \\
\hline WHR & $0.94(0.04)$ & $0.83(0.04)$ & $0.97(0.03)^{*}$ & $0.86(0.01)^{t+}$ \\
\hline Follow-up WHR & $0.95(0.04)$ & $0.84(0.03)$ & $0.99(0.05)^{* *}$ & $0.89(0.02)^{\dagger+}$ \\
\hline ASM (kg) & $25.03(6.11)$ & $18.45(2.48)$ & $27.33(2.46)$ & $18.72(2.24)$ \\
\hline Follow-up ASM (kg) & $25.11(2.66)$ & $18.36(1.78)$ & $28.07(2.94)^{* *}$ & $19.83(1.99)^{\dagger}$ \\
\hline TBF (kg) & $16.15(3.77)$ & $17.94(3.64)$ & $20.59(3.21)^{* *}$ & $22.22(2.55)^{\dagger}$ \\
\hline Follow-up TBF (kg) & $16.53(3.74)$ & $18.52(3.44)$ & $22.15(3.56)^{* *}$ & $25.27(2.08)^{+\dagger}$ \\
\hline ASM/TBF (\%) & $1.67(0.76)$ & $1.07(0.27)$ & $1.36(0.23)$ & $0.84(0.06)^{\dagger+}$ \\
\hline Follow-up ASM/TBF (\%) & $1.61(0.47)$ & $1.02(0.18)$ & $1.32(0.37)^{*}$ & $0.79(0.05)^{+\dagger}$ \\
\hline Albumin (g/L) & $45.15(2.59)$ & $45.44(2.45)$ & $44.71(3.02)$ & $46.94(2.03)$ \\
\hline Follow-up albumin (g/L) & $44.73(2.45)$ & $44.79(2.67)$ & $44.08(2.71)$ & $46.08(2.51)$ \\
\hline $\mathrm{DM}(n, \%)$ & 49 (19.6) & $38(22.1)$ & $3(20.0)$ & $1(11.1)$ \\
\hline
\end{tabular}

Continuous data were shown as mean (SD) and categorical data were $n(\%)$

Abbreviations: $B M I$ body mass index; WHR waist-hip ratio; $A S$ appendicular skeletal muscle mass; TBF total body fat; $D M$ diabetes mellitus Non obesity versus New obesity: Male: ${ }^{*} P<0.05,{ }^{* *} P<0.001$; Female: ${ }^{+} P<0.05,{ }^{++} P<0.001$

\section{Relationship between low baseline ASM/TBF and new obesity}

Logistic regression expounded that both men and women with decreased baseline ASM/TBF had higher risk of obesity over follow-up period ( $R R$ in model 6 were 5.664 for men and 34.856 for women, $95 \%$ confidence interval $(C I)$ in model 6 were $1.879-17.074$ and 3.930-309.153, separately) (Table 3).

\section{Relationship between longitudinal low ASM/TBF and new obesity}

There were 58 participants (13.0\%) with decreased ASM/TBF both at baseline and follow-up. Logistic regression showed that participants with decreased ASM/ TBF both at baseline and follow-up demonstrated higher risk of obesity ( $R R$ in model 9 were 9.299 for men and 69.637 for women, $95 \%$ CI in model 9 were 2.85030.341 and 7.200-673.552, respectively) (Table 4).

Table 2 Effect of baseline ASM/TBF on follow-up BMI

\begin{tabular}{llllll}
\hline & Male & & & Female & \\
\cline { 2 - 3 } & $\beta$ S SE) & $P$ value & & $\beta(S E)$ & $P$ value \\
\hline Model 1 & $-1.150(0.161)$ & $<0.001$ & & $-4.744(0.526)$ & $<0.001$ \\
Model 2 & $-1.141(0.159)$ & $<0.001$ & & $-4.748(0.526)$ & $<0.001$ \\
Model 3 & $-1.147(0.161)$ & $<0.001$ & & $-4.727(0.524)$ & $<0.001$
\end{tabular}

Abbreviations: SE standard error; $B M I$ body mass index; $A S M$ appendicular skeletal muscle mass; TBF total body fat

Model 1 was not adjusted for any factor. Model 2 was adjusted for age. Model

3 was adjusted for age, albumin, diabetes history

\section{Discussion}

This study reported for the first time that the usefulness of ASM/TBF in predicting for the risk of obesity, which was diagnosed by BMI, in elderly people.

The relationship between fat related indicators and obesity has been widely discussed in previous studies [16]. As BMI cannot distinguish fat mass and lean mass clearly and its prediction is prone to be intervened by the different adipose distribution, using BMI as a predictor for body fatness has been controversial [17]. WHR, another anthropometric index, is not universally acknowledged as an accurate indicator to identify individuals at risk for metabolic syndrome [18]. However, ASM/TBF, had its special advantages in considering functional and metabolic impacts of muscle aging and body fat deposition together.

The opinions of obesity were controversial in the elderly. One study involved elderly men in Australia, defined obesity as more than $30 \%$ body fat, showed that simple obesity was a protective factor for frailty [19]. But another prospective study demonstrated that obesity increased the mortality of incident cardiovascular diseases [20]. The protective role of obesity may be related to increased ASM, which was paralleled with increasing trends of TBF in the elderly. High ASM, especially robust lower limb strength, contributed to reducing falls [21]. In our study, we used BMI, which has been universally acknowledged as a golden standard, to diagnose obesity. Obesity people had higher ASM than non obesity regardless of gender, which verified the obesity 


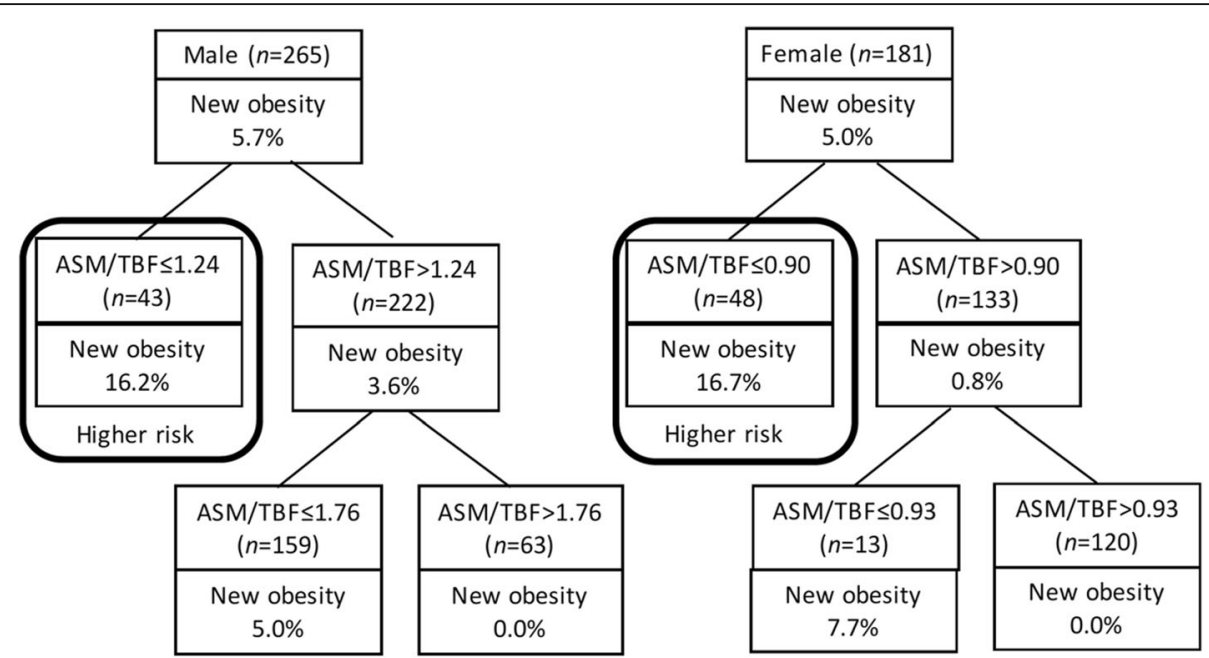

Fig. 1 Classification and regression tree models for new obesity on ASM/TBF. Abbreviation: ASM: appendicular skeletal muscle mass; TBF: total body fat

possibly positive role in older people. Moreover, both men and women with decreased baseline ASM/TBF had higher risk of obesity over follow-up period after adjusted for confounding factors, which further elaborated that ASM/TBF had a relationship with obesity. Thus gender imbalance was not an indispensable factor to ASM/TBF in predicting obesity.

Besides, there was no agreement about cut-off points for ASM/TBF to predict obesity. Jean Woo et al. [6] has reported that low ASM/TBFs were defined as less than 0.9 in men and 0.7 in women for 4-year physical limitation. Besides, 0.9 in men and 0.5 in women as cut-off points for 4-year slow walking speed were also shown in her study. However, in our study, CART was also used to determine baseline ASM/TBF cut-off points. The cutoff points of low ASM/TBF were defined as less than 1.24 in men and 0.90 in women, which meaned ASM/ TBF can be used as an indicator in predicting obesity.

From the view of histology and embryology, interestingly, skeletal muscle cells shared a common developmental origin with brown adipocytes, which broke down lipids to generate heat, thus reducing obesity [22]. The potential mechanisms of muscle to adipose tissue were chemical pathways. The bioactive substances produced from muscle affected adipose through endocrine or paracrine [23]. Thus, increased muscle mass had a protective function on overweight [24].

Even though it was a longitudinal study, the work had inevitable limitations. Firstly, as a single center study, its biases were unavoidable. For example, because of Berkson bias, a few number of elderly people aged over 80 was recruited in this study. So whether ASM/TBF was an universal and representative assessing indicator to older people needs further exploration. Secondly, visceral fat mass, which was a potential confounding variable, was not demonstrated in this study, therefore a large and longitudinal cohort is warranted to further demonstrate the relationship between visceral fat mass related indicators and obesity. Thirdly, because the new obese population is so small, the results from logistic
Table 3 Relationship between low baseline ASM/TBF and new obesity

\begin{tabular}{|c|c|c|c|c|}
\hline & \multicolumn{2}{|l|}{ Male } & \multicolumn{2}{|l|}{ Female } \\
\hline & $R R(95 \% \mathrm{Cl})$ & $P$ value & $R R(95 \% \mathrm{Cl})$ & $P$ value \\
\hline Model 4 & $5.201(1.777-15.226)$ & 0.003 & $26.400(3.205-217.479)$ & 0.002 \\
\hline Model 5 & $5.271(1.795-15.481)$ & 0.002 & 30.363 (3.618-254.852) & 0.002 \\
\hline Model 6 & 5.664 (1.879-17.074) & 0.002 & 34.856 (3.930-309.153) & 0.001 \\
\hline
\end{tabular}

Table 4 Relationship between longitudinal low ASM/TBF and new obesity

\begin{tabular}{|c|c|c|c|c|}
\hline \multirow[t]{2}{*}{ L-L } & \multicolumn{2}{|l|}{ Male } & \multicolumn{2}{|l|}{ Female } \\
\hline & $R R(95 \% \mathrm{Cl})$ & $P$ value & $R R(95 \% C l)$ & $P$ value \\
\hline Model 7 & $8.593(2.751-26.837)$ & $<0.001$ & $44.923(5.391-374.375)$ & $<0.001$ \\
\hline Model 8 & $8.444(2.693-26.477)$ & $<0.001$ & $55.377(6.399-479.241)$ & $<0.001$ \\
\hline Model 9 & $9.299(2.850-30.341)$ & $<0.001$ & $69.637(7.200-673.552)$ & $<0.001$ \\
\hline
\end{tabular}


regression may not be very reliable, which needs amplified sample size to further verify the predictive value of ASM/TBF in obesity.

\section{Conclusion}

Elderly people with decreased ASM/TBF had higher risk of new obesity, which suggested that ASM/TBF should be considered in obesity management in elderly people.

\section{Acknowledgements}

We would like to thank all participating patients.

\section{Competing of interest}

The authors declare no conflict of interest.

\section{Authors' contributions}

ZYJ participated in the design of the study and drafted the manuscript. FSH and $Y Y$ helped with the interpretation of data and the statistical analysis. LXY and WJX contributed to the conception of the study design and are the guarantors of the work. ZX contributed to the data collection process. All authors read and approved the final manuscript.

\section{Funding}

Not applicable.

\section{Availability of data and materials}

In attempt to preserve the privacy of patients, clinical data of patients will not be shared, but will be made publicly available upon request. If possible, ZYJ could be contacted once someone wants to request the data.

\section{Ethics approval and consent to participate}

The study protocol was approved by the Institutional Review Board of Chinese PLA General Hospital. All participants were given written informed consents to take part in the study.

\section{Consent for publication}

Not applicable.

\section{Author details}

'Department of Epidemiology, School of Public Health, Southern Medical University, Guangzhou, Guangdong, China. ${ }^{2}$ Department of Geriatric Cardiology, Chinese PLA General Hospital, Beijing, China. ${ }^{3}$ Department of Rehabilitation, Zhengzhou Central Hospital Affiliated to Zhengzhou University, Zhengzhou, China. ${ }^{4}$ Department of Rehabilitation, Chinese PLA General Hospital, Beijing, China. ${ }^{5}$ International Medical Center, Chinese PLA General Hospital, Beijing, China. ${ }^{6}$ Center for Healthy Aging and Development Studies, National School of Development, Peking University, Beijing, China. ${ }^{7}$ Institute of Geriatrics, National Clinical Research Center for Geriatric Diseases, Chinese PLA General Hospital, Beijing, China.

Received: 22 September 2019 Accepted: 29 March 2020

Published online: 19 April 2020

\section{References}

1. Lavie CJ, Deedwania P, Ortega FB. Obesity is rarely healthy. Lancet Diabetes Endocrinol. 2018;52213-8587:30143-8.

2. Mongraw-Chaffin M, Foster MC, Anderson CAM, Burke GL, Haq N, Kalyani RR, Ouyang P, Sibley CT, Tracy R, Woodward M, Vaidya D. Metabolically healthy obesity, transition to metabolic syndrome, and cardiovascular risk. J Am Coll Cardiol. 2018;71:1857-65.

3. Baik I. Forecasting obesity prevalence in Korean adults for the years 2020 and 2030 by the analysis of contributing factors. Nutr Res Pract. 2018;12: 251-7.

4. Carpenter CL, Yan E, Chen S, Hong K, Arechiga A, Kim WS, Deng M, Li Z, Heber D. Body fat and body-mass index among a multiethnic sample of college-age men and women. J Obes. 2013;2013:790654.

5. Xu T, Zhu G, Han S. Trend of body compositions with aging among Chinese adolescents, adults and elders. J Nutr Health Aging. 2015;19(10): 962-9.
6. Woo J, Leung J. Sarcopenic obesity revisited: insights from the Mr and Ms Os cohort. J Am Med Dir Assoc. 2018:S1525-8610:30106-13.

7. Zeng $Q$, Dong SY, Sun XN, Xie J, Cui Y. Percent body fat is a better predictor of cardiovascular risk factors than body mass index. Braz J Med Biol Res. 2012;45:591-600.

8. Zeng Q, Sun XN, Fan L, Ye P. Correlation of body composition with cardiac function and arterial compliance. Clin Exp Pharmacol Physiol. 2008;35:78-82.

9. Kohara K. Sarcopenic obesity in aging population: current status and future directions for research. Endocrine. 2014;45:15-25.

10. Zhang Y, Fu S, Wang J, Zhao X, Zeng Q, Li X. Association between geriatric nutrition risk index and low muscle mass in Chinese elderly people. Eur J Clin Nutr. 2019;73(6):917-23.

11. Zhang YJ, Fu SH, Wang JX, Zhao X, Zeng Q, Li XY. Predictive value of percentage body fat in aging people with low muscle mass: a 2.2-year longitudinal study. Arch Gerontol Geriatr. 2019:82:167-71.

12. Lohman TG, Roche AF, Martorell R. Anthropometric standardization reference manual(abridged edition). Champaign: Human Kinetics; 1988. p. 44-6.

13. Hu SS, Kong LZ, Gao RL, Zhu ML, Wang W, Wang YJ, Wu ZS, Chen WW, Liu $M B$; Editorial Board. Outline of the report on cardiovascular disease in China, 2010. Biomed Environ Sci. 2012;25(3):251-6.

14. Sub-Committee of Obesity of Chinese Society of Endocrinology of Chinese Medical Association. Chinese expert consensus on prevention and treatment of Obesity in adults. Chin J Endocrinol Metab. 2011;27:711-7.

15. Chinese Society of Diabetes. Chinese guidelines on prevention and treatment of type 2 diabetes (2013 edition). Chin J Diabetes Mellitus. 2014;6: 447-98.

16. Prado CM, Wells JC, Smith SR, Stephan BC, Siervo M. Sarcopenic obesity: a critical appraisal of the current evidence. Clin Nutr. 2012;31:583-601.

17. Lei SF, Liu MY, Chen XD, Deng FY, LV JH, Jian WX, Xu H, Tan LJ, Yang YJ, Wang YB, Xiao SM, Sun X, Jiang C, Guo YF, Guo JJ, Li YN, Liu YJ, Deng HW. Relationship of total body fatness and five anthropometric indices in Chinese aged 20-40 years: different effects of age and gender. Eur J Clin Nutr. 2006:60:511-8.

18. Perona JS, Schmidt-RioValle J, Rueda-Medina B, Correa-Rodríguez M, González-Jiménez E. Waist circumference shows the highest predictive value for metabolic syndrome, and waist-to-hip ratio for its components, in Spanish adolescents. Nutr Res. 2017:45:38-45.

19. Hirani V, Naganathan V, Blyth F, Le Couteur DG, Seibel MJ, Waite LM, Handelsman DJ, Cumming RG. Longitudinal associations between body composition, sarcopenic obesity and outcomes of frailty, disability, institutionalisation and mortality in community-dwelling older men: the Concord health and ageing in men project. Age Ageing. 2017;46:413-20.

20. Iliodromiti S, Celis-Morales CA, Lyall DM, Anderson J, Gray SR, Mackay DF, Nelson SM, Welsh P, Pell JP, Gill JMR, Sattar N. The impact of confounding on the associations of different adiposity measures with the incidence of cardiovascular disease: a cohort study of 296535 adults of white European descent. Eur Heart J. 2018;39:1514-20.

21. Flegal KM, loannidis JPA. The obesity paradox: a misleading term that should be abandoned. Obesity (Silver Spring). 2018;26:629-30.

22. Yang X, Bi P, Kuang S. Fighting obesity: when muscle meets fat. Adipocyte. 2014;3:280-9.

23. Kawao N, Kaji H. Interactions between muscle tissues and bone metabolism J Cell Biochem. 2015;116:687-95.

24. Vella CA, Cushman M, Van Hollebeke RB, Allison MA. Associations of abdominal muscle area and Radiodensity with Adiponectin and Leptin: the multiethnic study of atherosclerosis. Obesity (Silver Spring). 2018;26(7):1234-41.

\section{Publisher's Note}

Springer Nature remains neutral with regard to jurisdictional claims in published maps and institutional affiliations. 